\title{
Chemo-differentiating ABB' Multicomponent Reactions. Privileged Building Blocks
}

\author{
Authors - David Tejedor*ab and Fernando García-Tellado*ab \\ Receipt/Acceptance Data [DO NOT ALTER/DELETE THIS TEXT] \\ ${ }_{5}$ Publication data [DO NOT ALTER/DELETE THIS TEXT] \\ DOI: 10.1039/b000000x [DO NOT ALTER/DELETE THIS TEXT]
}

\begin{abstract}
Multicomponent reactions (MCRs) designated as $\mathrm{ABB}$ ' are defined as those reactions that introduce into the final product one molecule of component $\mathrm{A}$ and two molecules of component $\mathrm{B}$ in a chemo-differentiating manner. This ability to discriminate the incorporation of 10 component $\mathrm{B}$ ensures that these processes maintain the advantages of using multicomponent reactions in diversity-oriented molecular construction. Furthermore, they benefit from the fact that only two reagents need to be mixed together. Component $\mathrm{B}$ can be therefore considered to be a privileged building block, and the reactions in which it participates, chemo-differentiating multicomponent reactions. Among the reduced set of compounds capable of acting as such 15 building blocks, we discuss the use of ketenes, terminal conjugated alkynoates, enolisable carbonyl compounds, cyclic enol ethers and cyclic enamines.
\end{abstract}

\section{Introduction}

Although the availability of novel multicomponent reactions (MCRs) has increased dramatically over the last 20 decade, they cover a limited amount of the chemical space and much of it still remains to be discovered. ${ }^{1-9}$ There is no doubt that some of these transformations are important cornerstones in the diversity-oriented construction of molecular complexity due to their ability to incorporate, in a fast and efficient 25 manner, three or more components into a single product. This means that the structure of the product can be easily modified by small systematic variations of each of the starting materials. MCRs generally benefit from other aspects such as atom-economy, use of readily available starting materials, 30 resource effectiveness and bond-forming efficiency that render these reactions useful alternatives for the greener trend in which organic chemistry is engaged. In addition, because MCRs are one-pot processes with simpler experimental conditions that do not require the isolation of intermediates,

35 they are perfect candidates for combinatorial, automated synthesis and drug discovery.

Over the years, there have been various classification systems for MCRs, e.g. according to the components involved, the type of reaction or the reversibility of reactions 40 leading to intermediary products. In terms of the components involved, multicomponent reactions that utilise, for example, three components (3CR) can be divided into three different categories depending on the nature of the starting materials. $\mathrm{ABC}$ designates a multicomponent reaction involving three 45 different reagents; $\mathrm{ABB}$ ( or $\mathrm{AB}_{2}$ ), a reaction that involves one molecule of reagent $\mathrm{A}$ and two molecules of reagent $\mathrm{B}$ (Scheme 1, a); and finally, AAA, a reaction that involves three molecules of the same reagent. It appears reasonable that

\footnotetext{
${ }^{a}$ Instituto de Productos Naturales y Agrobiología, CSIC, Astrofísico Francisco Sánchez, 3, 38206 La Laguna, Tenerife, Canary Islands, Spain. Fax: int-34922-260135; Tel: int-34922-256847; E-mail:

fgarcia@ipna.csic.es; dtejedor@ipna.csic.es

$b$ Instituto Canario de Investigación del Cáncer, www.icic.es
}

in order to achieve the highest possible complexity and 50 diversity in the final products, the ideal multicomponent reaction would fall into the first category $(\mathrm{ABC})$ although this is not strictly necessary as evidenced by a few selected examples that have appeared in the literature over the last decade and that will be highlighted in this article. The key to 55 this particular category of multicomponent reactions that can be designated $\mathrm{ABB}$ ' is the chemo-differentiating incorporation of the component B in two distinct manners (B and B'), which ensures the complexity and functional diversity of the final product (Scheme 1, c). ${ }^{10} \mathrm{We}$ use the term chemo${ }_{60}$ differentiating to signify that each chemical function incorporated into the product is chemically different from the other. All of the selected examples share a common chemical property: they perform a chemical process able to transform a small set of reagents into a final product whose structure ${ }_{65}$ incorporates each reagent several times and in the form of differentiated chemical functions. In terms of diversity-

a)

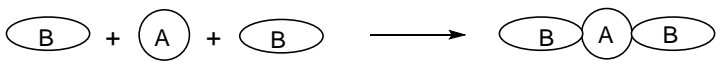

b)

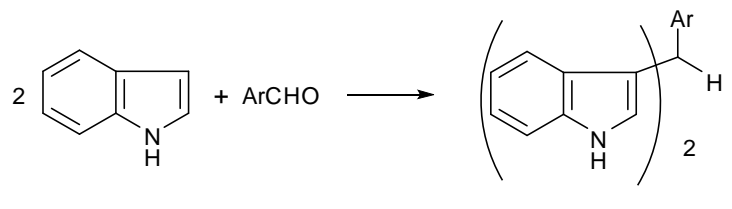

c)

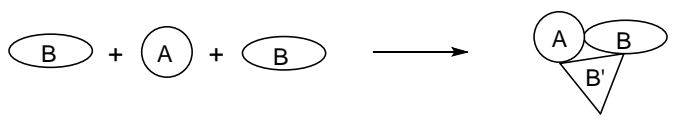

Scheme 1. a) $\mathrm{ABB}$ (or $\mathrm{AB}_{2}$ ) 3CR. Both molecules of component $\mathrm{B}$ are incorporated into the product in a similar manner. b) Example of an $A B B$ 3CR. Synthesis of bis(indolyl)methanes from indole and aromatic aldehydes. c) ABB' 3CR. Two molecules of component B are introduced into the product as different building blocks. The product gains structural complexity and diversity. 
generation, it means that two identical starting units construct highly functionalized products containing a broader set of chemical functionalities or structural motifs. Furthermore, 70 there are two more beneficial aspects that arise from the fact that only two reagents need to be mixed together: (a) the reaction processing becomes simpler, saving time and equipment, and (b) since one of the drawbacks of MCRs is that all the starting materials must tolerate the initial reaction 75 conditions, obviously, there is a greater possibility that only two starting materials tolerate those conditions rather than three.

The chemical realization of this design concept is contingent on two factors. First, the construction of a suitable 80 chemo-differentiating reaction network, and second, the chemical availability of the suitable starting reagents. This ability is denoted only by a short number of chemical functionalities. We refer to them as privileged building blocks to highlight this reactivity-based property (the term privileged 85 is used to signify an advantageous reactivity profile more than a universal one). Among the reduced set of compounds displaying this particular reactivity profile, we will highlight the use of ketenes, terminal conjugated alkynoates, enolisable carbonyl compounds, cyclic enol ethers and cyclic enamines.

90 The examples that have appeared in the literature are not all three-component chemo-differentiating MCRs. Following the same reasoning that we have applied to 3C MCRs, other examples involving more components can be easily catalogued into new categories such as AABB', ABB'C, etc.

${ }_{95}$ Examples falling into some of these more complex categories are scarcer and they will also be discussed in this review.

Finally, many of the reactions appearing in this article had not been originally presented as multicomponent reactions and this has made it more difficult to search for them in keyword 100 databases. Although a careful inspection has been conducted to find examples which would reflect the objectives of this review, it does not intend to be fully comprehensive, but rather, a conceptual overview of a selected group of examples which will most likely aid in the search for new MCRs and 105 that will benefit both academic and industrial scientists.

\section{Ketenes}

It has been known for some time that ketenes react with isocyanides in an $\mathrm{ABB}$ (or $\mathrm{AB}^{2}$ ) 3CR to form 2,5110 dialkylidene-4-imino-1,3-dioxolane derivatives ${ }^{11} 2$ (Scheme 2). Although in this reaction three new bonds and one ring are formed, it appears obvious from observing the structure of the product that the ketene building blocks contribute limited functional diversity to the final product. This occurs because 115 both ketenes are incorporated following similar nucleophilic additions. The first one involves the addition of the initial isocyanide and the second one the enolate of the zwitterionic intermediate $\mathbf{1}$.

A very recent example shows the versatility of ketenes as 120 privileged building blocks (Figure 1), and at the same time allows us to show again the difference between an ABB MCR and a chemo-differentiating ABB' MCR.

Robertson et al. have recently shown ${ }^{12}$ that under controlled experimental conditions (low practical concentrations of

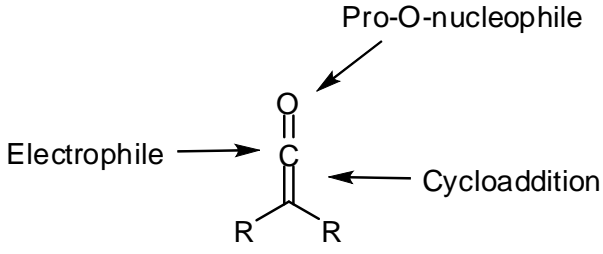

Figure 1. Reactivity profile of ketenes

125 ketene and presence of $\mathrm{TMSCl}$ ), intermediate $\mathbf{1}$ can rearrange and, more importantly, undergo a $[2+2]$ cycloaddition with another ketene molecule to give the more elaborated product 4 as a mixture of two diastereomers (Scheme 2). The increased structural complexity and diversity are mainly a result of the 130 new $\mathrm{ABB}$ ' 3CR in which the ketene building blocks are introduced into the product in two different manners: via a nucleophilic addition and a cycloaddition. The mechanism proposed by the authors includes the electrocyclisation of zwitterion $\mathbf{1}$ in the absence of a high concentration of 135 diphenylketene, tautomerisation and the successive $[2+2]$ cycloaddition that generates the $\beta$-lactam ring. Key to the formation of product 4 , instead of 1,3-dioxolane derivative 2 , is the slow addition of the diphenylketene to avoid its intermolecular reaction with intermediate 1. Overall, this 140 process generates four new bonds and two rings in a single operation.

A further modification in the experimental conditions, which includes an additional equivalent of diphenylketene, brings about the transformation of $\mathbf{4}$ into product $\mathbf{5}$ via an ene 145 reaction. In this new $\mathrm{ABBB}^{\prime} 4 \mathrm{CR}$, the additional ketene

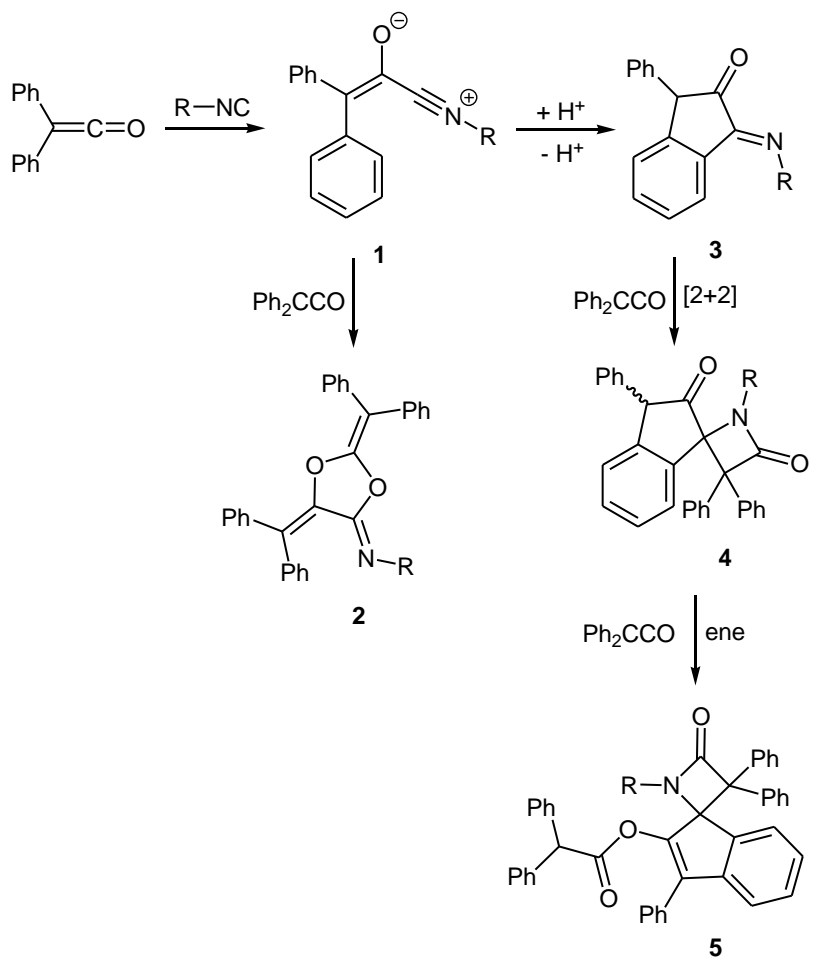

Scheme 2. Ketene-based ABB' 3CR 
building block is also introduced into the final product through a nucleophilic addition, generating an additional carbon-oxygen bond and ester functionality. BBB' denotes therefore that only two out of the three type B building blocks 150 are incorporated in a similar manner.

\section{Terminal Conjugated Alkynoates}

Terminal conjugated alkynoates are small molecules with an extremely high degree of chemical complexity (Figure 2).

155 These molecules can react with a wide range of reagents that include for example nucleophiles, strong bases, dienes or dipoles.

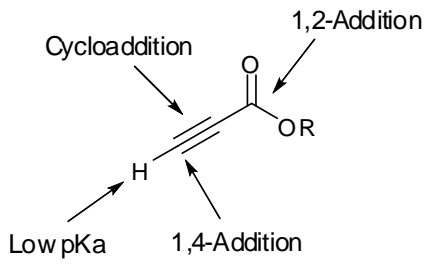

Figure 2. Reactivity profile of terminal conjugated alkynoates

Our own contributions to this chemistry combine the two main chemical properties of terminal conjugated alkynes: their 160 relatively high acidity $(\mathrm{pKa}<18.8)^{13}$ and their good Michaelacceptor character. ${ }^{14}$ Interestingly, this means that this privileged building block can act as a nucleophile and an electrophile in the same MCR. Depending on the catalyst and the reaction conditions, one molecule of an aliphatic aldehyde 165 or certain $\alpha$-dicarbonyl compounds and two molecules of a terminal conjugated alkynoate may be combined to yield enolprotected propargylic alcohols 6 (Scheme 3) or 1,2dihydrofuran derivatives 12 (Scheme 4). ${ }^{15,16}$

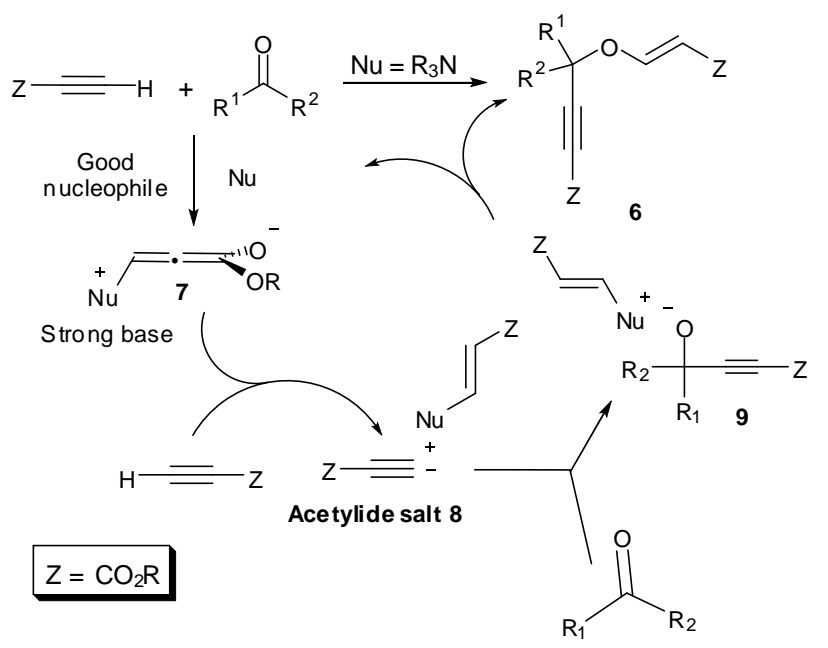

Scheme 3. Conjugated acetylide-driven ABB' 3CR.

Both processes represent examples of $\mathrm{ABB}$ ' 3CRs and have 170 a common starting point. The energetically favoured nucleophilic addition on the terminal conjugated alkyne generates the zwitterionic allenolate 7 , which deprotonates the starting conjugated alkyne to generate the reactive acetylide salt 8. Overall, a catalytic amount of a good nucleophile
175 generates a catalytic amount of a strong base. Once formed, the reactive acetylide salt $\mathbf{8}$ adds to an electrophile present in the reaction medium to give the expected addition products. Aldehydes or $\alpha$-dicarbonyl compounds bearing no protons more acidic than the terminal alkynoate itself are good 180 electrophiles and their adducts, propargylic alkoxides 9, are themselves good nucleophiles to give Michael addition on either the reactive conjugated alkene counterion affording enol-protected propargylic alcohol derivatives $\mathbf{6}$, or a free starting terminal conjugated alkynoate affording the 185 corresponding 1,2-dihydrofuran 12. The formation of heterocyclic compound $\mathbf{1 2}$ is the result of an intramolecular Michael addition in intermediate $\mathbf{1 0}$ and the protonation of $\mathbf{1 1}$ by the starting alkynoate. As a result this $\mathrm{MCR}$ generates three new bonds (two $\mathrm{C}-\mathrm{C}$ and one $\mathrm{C}-\mathrm{O}$ ) and one ring.
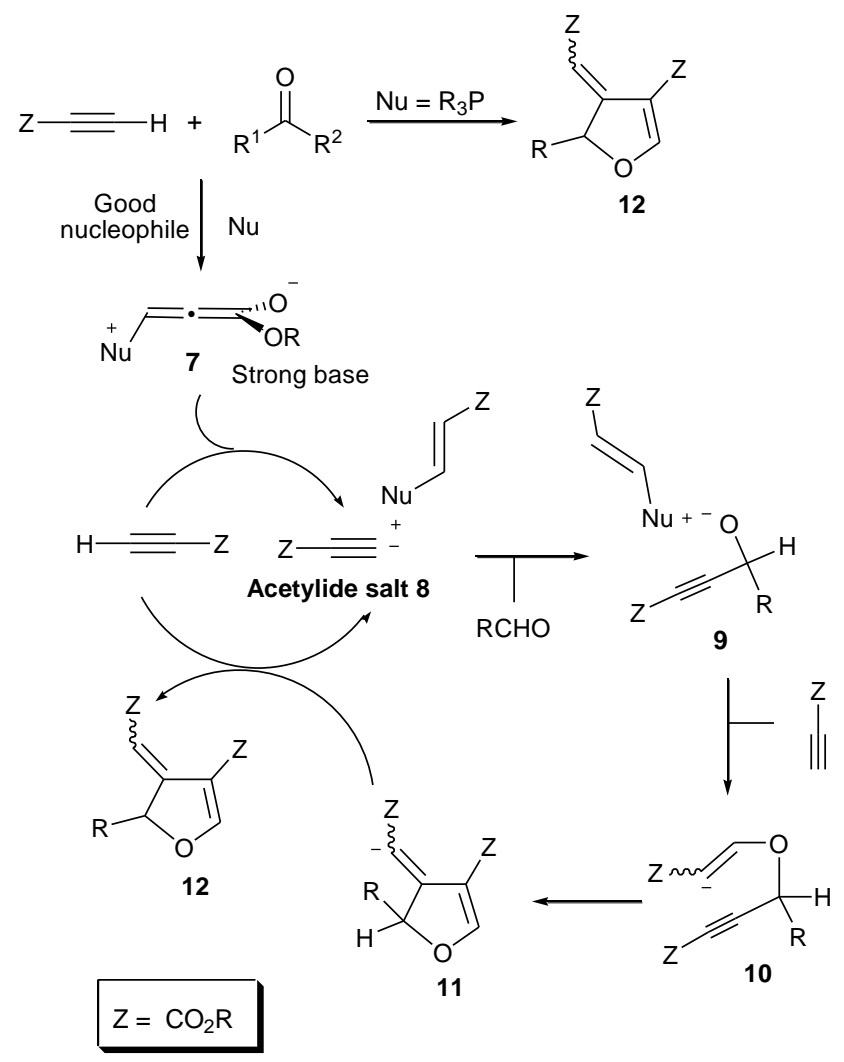

Scheme 4. Conjugated acetylide-driven ABB' 3CR.

190 Interestingly, the nucleophilic catalyst plays at least two important roles. It not only triggers the acetylide generation, but it is also crucial in determining the outcome of the reaction. Under the appropriate reaction conditions, tertiary amines funnel the process towards $\mathbf{6}$ while tertiary phosphines 195 afford 12. In both cases, one building block is introduced as an acetylide ion (nucleophile) due to its increased acidity and one as a Michael-acceptor (electrophile).

Finally, as it can be anticipated by the use of multicomponent reactions, the products (6 and 12) constitute 200 highly functionalized units which are well suited for using as scaffolds for further diversity-oriented molecular construction. In this regard we have already explored some of 
the possibilities of product 6 in the efficient synthesis of 1,3oxazolidines ${ }^{17}$ and polysubstituted pyrroles. ${ }^{18}$ The simplicity 205 of the first ABB' MCR, coupled with another domino process has allowed us to build small libraries of compounds in a programme aimed at the discovery of bioactive substances for cancer treatment. ${ }^{19,20}$

More recently, a similar-in-origin triethylamine-catalyzed 210 AABBB' type 5CR of an alkyl propiolate with aromatic aldehydes has been described. ${ }^{21}$ This temperature-dependent sequence of cascade reactions generates, at a first stage, 2,3,9,9a-tetrahydronaphtha[2,3-b] furans $\mathbf{1 3}$ with an impressive bond-forming efficiency of seven new bonds (five ${ }_{215} \mathrm{C}-\mathrm{C}$ and two $\mathrm{C}-\mathrm{O}$ ) in a single operation (Scheme 5). Upon warming, these products can be selectively transformed into naphthalene derivatives $\mathbf{1 4}$ or $\mathbf{1 5}$. The mechanism proposed by the authors involves the formation of the enol-protected propargylic alcohol 6 (already described in Scheme 3) which 220 undergoes a further acetylide addition to generate an intermediate that is able to react with another molecule of the initial aldehyde. This is followed by a series of rearrangements and protonation to afford 13. The cascade of reactions has been able to incorporate two molecules of 225 aromatic aldehydes and three molecules of ethyl propiolate into the product. Both aldehyde units are incorporated

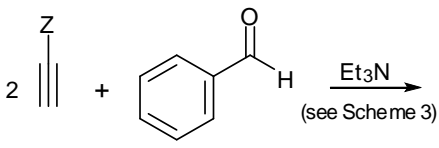<smiles>[Z]C#CC(OC=C[Z])c1ccccc1</smiles><smiles>[Z]C#CC(=C([Z])C([18O])OCC)C(OC=C[Z])c1ccccc1</smiles><smiles></smiles><smiles>[Z]C#CC(=C[Z])C(OC=C[Z])c1ccccc1</smiles>

C-H addition Michael addition<smiles></smiles><smiles>[Z]C=COC1=C2C([Z])C([Al])OC2C([Z2])c2ccccc21</smiles><smiles>[Z]C=COc1c(C[Z])cc([Z])c2ccccc12</smiles>

15
$\mathrm{Et}_{3} \mathrm{~N}, \Delta$ Retro-aldol addition<smiles>[Z]c1cc(C(C)C([Y])O)c(O)c2ccccc12</smiles>

Scheme 5. Conjugated acetylide-driven AABBB' 5CR.

following similar 1,2-additions on the carbonyl moiety (AA) but the ethyl propiolate units are incorporated in two different ways: two as sources of acetylide nucleophiles and one as a 230 Michael-acceptor (BBB').

\section{Enolizable carbonyl compounds}

There are two well-known reactivity sites in this group of compounds (Figure 3 ). The increased acidity of $\alpha$-hydrogens, 235 which leads to the formation of enolate ions, and the electrophilicity of the carbonyl group give rise to the aldol reaction. The corresponding $\beta$-hydroxycarbonyl compound (or the $\alpha, \beta$-unsaturated derivative) can subsequently be used in a further reaction to give a more elaborated product. When both 240 reactions are combined in a one-step process, the bases are obtained for an ABB' type MCR. Not surprisingly, there are a considerably higher number of examples that include the use of enolisable carbonyl compounds as the privileged building blocks.
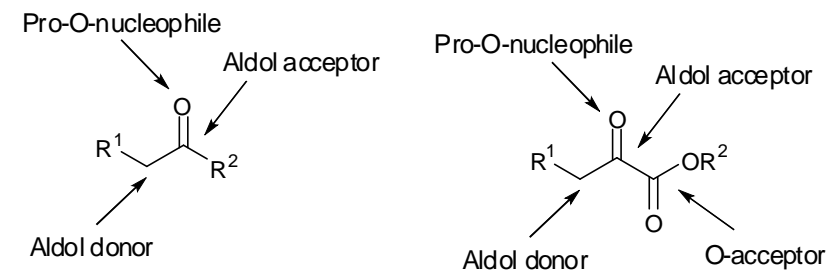

Figure 3. Reactivity profiles of simple enolisable carbonyl compounds and enolisable $\alpha$-ketoesters

245 The first two examples show the reaction of ketones with aromatic amines. The group of Theoclitou has recently developed an ABB' 3C synthesis of polysubstituted 1,2dihydroquinolines $\mathbf{1 6}$ via a modified Skraup reaction that uses ketone and the desired aniline as the starting materials 250 (Scheme 6). ${ }^{22}$ This $\mathrm{Sc}(\mathrm{OTf}) 3$-catalysed reaction proceeds under much milder conditions than the original Skraup cyclisation (at $145{ }^{\circ} \mathrm{C}$ under pressure for 2-3 days in the presence of iodine), while allowing to expand the scope of the substituents in both the aromatic amine and the ketone.

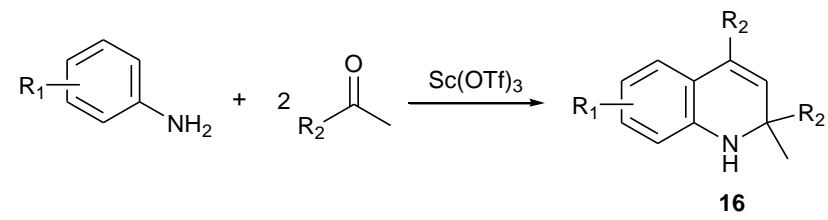

Scheme 6. Sc-catalyzed ABB' 3CR synthesis of dihydroquinolines.

When $o$-phenylenediamine is used as the aromatic amine, the result is the formation of 1,5-benzodiazepine derivatives 17 via another $\mathrm{ABB}$ ' $3 \mathrm{CR}$ as has been described by various groups under a wide range of different reaction conditions 260 (Scheme 7). ${ }^{23}$ Aliphatic ketones (cyclic and acyclic), and substituted acetophenones have all been used as the privileged building blocks in these transformations. The originally proposed mechanism ${ }^{24}$ involves the formation of the $\alpha, \beta$ unsaturated carbonyl compound followed by two consecutive 265 additions (one Michael addition and one 1,2-addition) of the 
<smiles></smiles><smiles>[R]C/C=C(/[R])C(=O)CC</smiles>

Scheme 7. ABB' 3C synthesis of 1,5-benzodiazepine derivatives.

amino groups on the aldol intermediate. The number of catalysts used to promote this reaction is large and includes Lewis and protic acids, microwave conditions, use of ionic liquids and solid supports.

270 There are other examples showing $3 \mathrm{C}$ or even $4 \mathrm{C}$ chemodifferentiating MCRs of aldehydes with either amides or aliphatic amines. Interestingly, the reaction of aldehydes and amides can give a number of products depending on the reaction conditions. The simplest case is the ABB' 3-C 275 trifluoromethanesulfonic acid-catalyzed condensation of a primary amide with two equivalents of an aldehyde to give the corresponding $\beta$-amido aldehyde $\mathbf{1 8}$ as a mixture of two diastereomers (Scheme 8). ${ }^{25}$ The authors propose the in situ formation of the imine from an amide and an aldehyde, 280 followed by an amidoalkylation with a second molecule of aldehyde. Experiments with $\alpha, \beta$-unsaturated aldehydes and amides under the same reaction conditions did not lead to the expected product, ruling out the possibility of a direct aldol condensation followed by dehydration and conjugate addition.

285 The $\beta$-amido aldehydes obtained are of synthetic value because they are relatively stable derivatives of $\beta$-amino aldehydes which are more difficult to isolate. It should also be added that these authors developed a similar methodology under different acidic conditions with the use of acetals as the 290 source of the corresponding aldehydes. ${ }^{26}$<smiles>[R]CC=CC(=O)O</smiles><smiles>[R7]CC(NC([R])=O)C([R])C=O</smiles>

Scheme 8. Trifluoromethanesulfonic acid-catalyzed ABB' 3-C synthesis of $\beta$-amido aldehydes.

More appealing seems the ABB'C 4C coupling of amides, aldehydes and dienophiles (scheme 9). ${ }^{27}$ Beller et al. further developed this methodology, which involves the Diels-Alder reaction of a 1-acylamino-1,3-diene intermediate 19 with<smiles>[Z]C#CC[R]([H])=C1C=CC(=O)O1</smiles>

Scheme 9. ABB'C $4 \mathrm{C}$ coupling of amides, aldehydes and dienophiles 
295 different dienophiles (dialkyl acetylenedicarboxylates, acrylonitrile, maleic anhydride or maleimides), to efficiently prepare highly functionalized cyclohexene and cyclohexadiene derivatives $\mathbf{2 0 - 2 3}$ in a diastereoselective manner. Remarkably, although up to four stereogenic centres 300 are created, the authors only observe the formation of one diastereomer in each case. This reaction features the formation of four new bonds (three carbon-carbon and one carbon-nitrogen) and one new ring. Additionally, when maleic anhydride is used as the dienophile, the expected 305 product undergoes a rearrangement through a subsequent intramolecular amidation of one carboxylic moiety to give bicyclic products 22. The key to this MCR (coined by the authors as the AAD reaction: amide-aldehyde-dienophile) is that although there are numerous side reactions which could 310 likely proceed under these conditions, there is a selective formation of the 1-acylamino-1,3-diene intermediate 19 which is trapped by the appropriate dienophile.

Similarly, the group of Ishii reported the preparation of polysubstituted pyrroles $\mathbf{2 4}$ via a samarium trichloride $315\left(\mathrm{SmCl}_{3}\right)$-catalyzed ABB'C 4-C coupling of amines, aldehydes and nitroalkanes (Scheme 10). ${ }^{28}$ As before, the aldehyde acts as the privileged building block in the formation of a key $\alpha, \beta$ unsaturated imine intermediate 25. Addition of the nitroalkane to 25 is followed by a rearrangement and elimination of $\mathrm{H}_{2} \mathrm{O}$ 320 and HNO to give the pyrrole $\mathbf{2 4}$. The authors clearly show that the only role of the catalyst is to form the $\alpha, \beta$-unsaturated imine because, in a separate experiment, the coupling of a preformed $\alpha, \beta$-unsaturated imine with a nitroalkane takes place in the absence of $\mathrm{SmCl}_{3}$ to form the expected product.

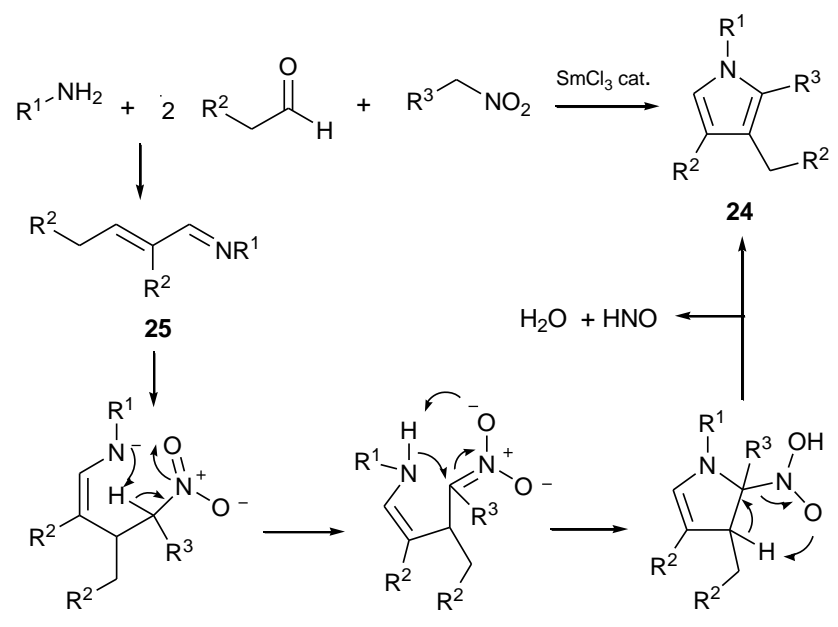

Scheme10. $\mathrm{SmCl}_{3}$-catalyzed $\mathrm{ABB}$ ' $\mathrm{C} 4 \mathrm{CR}$ of amines, aldehydes and nitroalkanes.

325 The last example in this category of enolisable carbonyl compounds also comes from our laboratory and is based on the ABB' $3 \mathrm{C}$ organocatalyzed homoaldolic condensation of $\alpha$ ketoesters (Figure 3 ) in the presence of terminal conjugated alkynoates. ${ }^{29}$ In general, $\alpha$-ketoesters bearing $\alpha$-hydrogens are 330 more reactive and acidic than simple aldehydes and ketones. Since they are even more acidic than terminal alkynoates, their combined reaction catalyzed by a nucleophile (tertiary amine) triggers a cascade of reactions that starts with a selfcondensation of the $\alpha$-ketoester, followed by a lactonisation 335 and Michael addition to afford isotetronic acids 26 (Scheme 11). It is remarkable that one of the $\alpha$-ketoester units acts twice as the nucleophile (via the $\alpha$-carbon in the aldol condensation and via the oxygen in the lactonisation) and the other $\alpha$-ketoester unit twice as the electrophile (the ketone 340 group in the aldol condensation and the ester group in the lactonisation). The role of triethylamine is to indirectly trigger the aldol reaction. As previously seen in Scheme 3, $\mathrm{Et}_{3} \mathrm{~N}$ adds to an alkyl propiolate to generate a much stronger base 7 which is the species that deprotonates the first $\alpha$-ketoester in 345 the self-condensation.

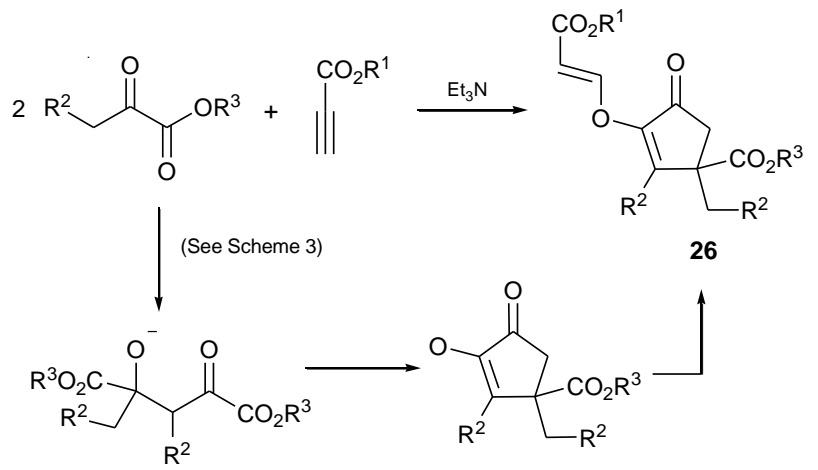

Scheme 11. Homoaldol-based ABB' 3CRs.

\section{Cyclic enol ethers or enamines}

3,4-Dihydro-2H-pyran, 2,3-dihydro-2H-furan, N-protected 2,3-dihydropyrroles, and N-protected 1,2,3,4350 tetrahydropyridines (Figure 4) have all been used as privileged building blocks in ABB' type MCRs when combined with aryl primary amines.

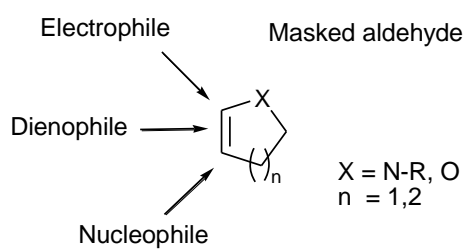

Figure 4. Reactivity profile of cyclic enol ethers or enamines

These substrates can act as the aldehyde component in the 355 in situ formation of the corresponding imines and as electronrich dienophiles in a subsequent aza-Diels-Alder reaction (Scheme 12). In consequence, a tricyclic product 27 with a tetrahydroquinoline core is efficiently obtained.

Although Povarov and Michailov published in 1964 the 360 first MCR of a substituted aniline with an enol ether, ${ }^{30}$ it was Batey in $1999^{31}$ who realized the full scope of this reaction and the dual role played by one of the components. Numerous studies have since appeared regarding the reaction medium, the substrates, the nature of the catalyst and the 365 diastereoselectivity of the process. ${ }^{32-36}$ 


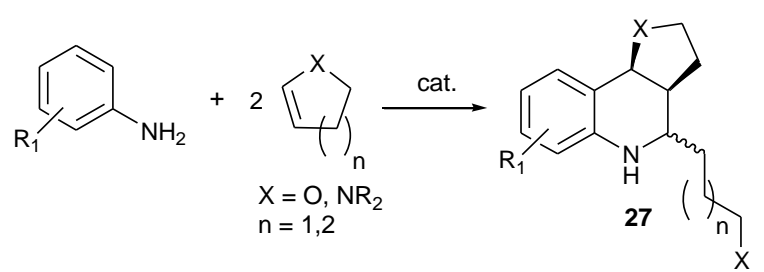

Scheme 12. ABB' $3-\mathrm{C}$ synthesis of the tetrahydroquinoline core

The use of cyclic enol ethers has been more common than their nitrogen counterparts. Batey et al. first reported the $\mathrm{Dy}(\mathrm{OTf})_{3}$-catalyzed reaction of 2,3-dihydro- $2 \mathrm{H}$-furan with 370 various substituted anilines to form the corresponding 2(hydroxyalkyl)tetrahydroquinoline derivatives $27(\mathrm{X}=0, \mathrm{n}=$ 1). ${ }^{19}$ More recently, further research has shown that the reaction can be performed with $\mathrm{KSF}$ clay, $\mathrm{InCl}_{3}$ in $\mathrm{H}_{2} \mathrm{O}$, $\mathrm{Sc}(\mathrm{OTf})_{3}$ in [bmim]PF6, In in aqueous $\mathrm{HCl}$, and without 375 catalyst in hexafluoroisopropanol. Although more scarce, the use of endocyclic enamines has found a relevant application because the reaction of methyl 4-aminobenzoate with $\mathrm{N}$ protected 2,3-dihydropyrrole provides direct access to the core structure of the Martinella alkaloids (Figure 5).

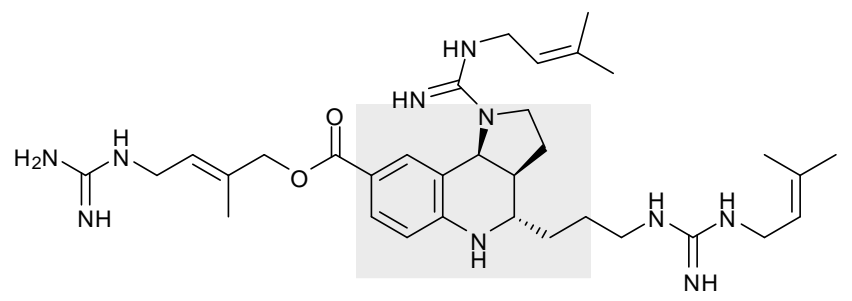

(+)-martinelline

Figure 5

380

Martinelline and martinellic acid are natural products found in root extracts of the Amazonian Martinella iquitosensis vine which has been used by the indigenous population to treat various eye ailments. This MCR allows the rapid construction 385 of the tricyclic core of these guanidine alkaloids with the correct diastereoselectivity in only two synthetic steps and good overall yield, and Batey et al. have already accomplished its total synthesis using this MCR as the key step. ${ }^{34}$ Essential to their success was the search for the catalyst that would 390 favour the desired endo adduct during the hetero Diels-Alder reaction. Ultimately, camphorsulfonic acid in THF was chosen to predominately form the correct diastereomer.

\section{Summary and outlook}

395 We have shown how the chemo-differentiated incorporation of identical building blocks into the final product can transform a bimolecular reaction into a MCR. These reagents can be considered to be privileged building blocks because they have the ability to transform one chemical functionality 400 in the starting material into a broader set of functionalities or structural motifs in the product. This ensures that the MCRs in which they participate maintain the ability to generate molecular complexity and diversity.

The ABB' notation ${ }^{10}$ is proposed to designate the chemo405 differentiating $3 \mathrm{C} \mathrm{MCR}$ that introduces into the final product one molecule of component $\mathrm{A}$ and two molecules of component B. Most importantly, this notation highlights the dual role played by component $\mathrm{B}$ and it stresses that it is incorporated in two different manners. Although ABB' $3 \mathrm{C}$ ${ }_{410}$ MCRs are the most common among this type of transformations, we have also shown more complex multicomponent reactions such as $\mathrm{ABBB}^{\prime} 4 \mathrm{C}, \mathrm{ABB}^{\prime} \mathrm{C} 4 \mathrm{C}$ and AABBB' $5 \mathrm{C}$ reactions.

Among the reduced set of compounds that fulfil the needed 415 requirements, we have highlighted the use of ketenes, terminal conjugated alkynoates, enolisable aldehydes or ketones, and cyclic enol ethers or enamines. Although these building blocks have appeared in the literature more frequently in this type of reactions, these are not the only reagents that meet the 420 needed requirements and it is anticipated that in the future we will see a larger and broader set of such chemo-differentiating multicomponent reactions that will possibly lead to novel molecular frameworks in a single reaction step.

Finally, we hope that in the future, when new reactions of 425 this type appear in the literature, they will be categorized as chemo-differentiating reactions in order that they can enter the pool of MCRs available to organic chemists.

\section{Acknowledgements}

430 The research of this group is supported by the Spanish Ministerio de Educación y Ciencia and the European Regional Development Fund (CTQ2005-09074-C02-02), and the Instituto Canario de Investigación del Cáncer (ICIC-GI no. 10/2005; ISCiii, RTICCC C03/10). D. T. is the recipient of a 435 postdoctoral I3P fellowship from the Consejo Superior de Investigaciones Científicas.

\section{Notes and references}

1. Multicomponent Reactions, eds. J. Zhu, H. Bienaymé, WilleyVCH: Weinheim, Germany, 2005.

2. For recent developments in isocyanide-based MCRs in applied chemistry, see: A. Dömling, Chem. Rev. 2006, 106, 17-89.

3. For asymmetric MCRs, see: D. J Ramón,. M. Yus, Angew. Chem. Int. Ed. 2005, 44, 1602-1634.

4. For the 1,3-Dicarbonyl derivatives-based MRs, see : C. Simon, T. Constantieux, J. Rodriguez, Eur. J. Org. Chem. 2004, 49574980.

5. For amidocarbonylation-based MCRs, see: A. Jacobi von Wangelin, H. Neumann, D. Gördes, S. Klaus, D. Strübing, M. Beller, Chem.-Eur. J. 2003, 9, 4286- 4294.

450 6. For recent developments in the isonitrile-based multicomponent synthesis of heterocycles, see: J. Zhu, Eur. J. Org. Chem. 2003, 1133-1144.

7. For recent advances in solution-phase multicompont methodology for the synthesis of heterocycles, see: R. V. A. Orru, M. de Greef, Synthesis 2003, 10, 1471-1499.

8. For a concept article on the synthetic efficiency associated with MCRs, see: H. Bienaymé, C. Hulme, G. Oddon, P. Schmitt, Chem.-Eur. J. 2000, 6, 3321-3328.

9. For a review on the discovery of new MCRs with combinatorial methods, see: L. Weber, K. Illgen, M. Almstetter, Synlett 1999, 366-374.

10. R. A. Batey et al. have previously suggested both $A B B$ and ABB'notations to highlight the dual role played by component 
$\mathrm{B}$ in a $3 \mathrm{C} \mathrm{MCR} .{ }^{34,35}$ We believe that the $\mathrm{ABB}$ ' notation reflects unambiguously the dual role played by component $\mathrm{B}$.

11. I. Ugi, K. Rosendhal, Chem. Ber. 1961, 94, 2233.

12. J. Robertson, S. J. Bell, A. Krivokapic, Org. Biomol. Chem. 2005, 3, 4246-4251.

13. J. Kresge, P. Pruszynski, J. Org. Chem. 1991, 56, 4808-4811.

14. P. Perlmutter, in Conjugated Addition Reactions in Organic Synthesis, Pergamon Press: Oxford, 1992.

15. D. Tejedor, F. García-Tellado, J. J. Marrero-Tellado, P. de Armas, Chem-Eur. J. 2003, 9, 3122-3131.

16. P. de Armas, F. García-Tellado, J. J. Marrero-Tellado, D. Tejedor, M. A. Maestro, J. González-Platas, Org. Lett. 2001, 3, 1905-1908.

17. D. Tejedor, A. Santos-Expósito, D. González-Cruz, F. GarcíaTellado, J. J. Marrero-Tellado, J. Org. Chem. 2005, 70, 10421045.

18. D. Tejedor, D. González-Cruz, F. García-Tellado, J. J. Marrero-Tellado, M. L. Rodríguez, J. Am. Chem. Soc. 2004, 126, 8390-8391.

19. J. M. Padrón, D. Tejedor, A. Santos-Expósito, F. GarcíaTellado, V. S. Martín, J. Villar, Bioorg. Med. Chem. Lett. 2005, 15, 2487-2490.

20. J. M. Padrón, D. Tejedor, A. Santos-Expósito, F. GarcíaTellado, J. Villar, V. S. Martín, Lett. Drug. Des. Disc. 2005, 2, 529-532.

490

21. Y.-G. Wang, S. -L. Cui, X .-F. Lin, Org. Lett. 2006, 8, 12411244.

22. M.-E. Theoclitou, L. A. Robinson, Tetrahedron Lett. 2002, 43, 3907-3910 and references cited therein.

23. R. Varala, R. Enugala, S. Nuvula, S. R. Adapa, Synlett 2006, 1009-1014 and references cited therein.

24. J. A. L. Herbert, H. Suschitzky, J. Chem. Soc., Perkin Trans. 1, 1974, 2657-2661.

25. C. M. Marson, A. Fallah, Chem. Commun. 1998, 23, 83-84.

26. C. M. Marson, S. Pucci, Tetrahedron Lett. 2004, 45, 90079010.

27. H. Neumann, A. J. von Wangelin, D. Gördes, A. Spannenberg, M. Beller, J. Am. Chem. Soc. 2001, 123, 8398-8399.

28. H. Shiraishi, T. Nishitani, S. Sakaguchi, Y. Ishii, J. Org. Chem. 1998, 63, 6234-6238.

29. D. Tejedor, A. Santos-Expósito, F. García-Tellado, Chem. Commun. 2006, 2667-2669.

30. L. C. Povarov, B. M. Michailov, Izv. Akad. Nauk. SSSR, Ser. Khim. 1964, 12, 2221.

31. R. A. Batey, P. D. Simoncic, D. Lin, R. P. Smyj, A. J. Lough, Chem. Commun. 1999, 651-652.

32. L. Chen, Z. Li, C.-J. Li, Synlett 2003, 5, $732-734$ and references cited therein.

33. Di Salvo, M. V. Spanedda, M. Ourévitch, B. Crousse, D. Bonnet-Delpon, Synthesis 2003, 14, 2231-2235.

34. D. A. Powell, R. A. Batey, Tetrahedron Lett. 2003, 44, 75697573.

35. D. A. Powell, R. A. Batey, Org. Lett. 2002, 4, 2913-2916 and references cited therein.

36. J. S. Yadav, B. V. S. Reddy, V. Suniths, K. S. Reddy, K. V. S. Ramakrishna, Tetrahedron Lett. 2004, 45, 7947-7950 and references cited therein. 


\section{Single column figure/scheme (below)}

$X$

Fig./Scheme XX Caption.

\section{Double column figure/scheme (below)}

$$
\mathrm{X}
$$

Fig./Scheme XX Caption. 